\title{
The Association between Expression of NK Cells and Prognosis of Patients with HBV Acute-on-Chronic Liver Failure in Advanced Phase
}

\author{
Weizhen Weng1, Yiming Shi², Xiaohua Peng³, Jing Xiong1, Huijuan Cao', Bingliang Lin ${ }^{*}$ \\ ${ }^{1}$ Department of Infectious Diseases, the Third Affiliated Hospital of Sun Yat-Sen University, Guangzhou, China \\ ${ }^{2}$ Department of Intensive Care Unit, the Sixth Affiliated Hospital of Sun Yat-Sen University, Guangzhou, China \\ ${ }^{3}$ Department of Gastroenterology, the Seventh Affiliated Hospital of Sun Yat-Sen University, Shenzhen, China \\ Email: *lamikin@126.com
}

How to cite this paper: Weng, W.Z., Shi, Y.M., Peng, X.H., Xiong, J., Cao, H.J. and Lin, B.L. (2017) The Association between Expression of NK Cells and Prognosis of Patients with HBV Acute-on-Chronic Liver Failure in Advanced Phase. Advances in Infectious Diseases, 7, 118-125.

https://doi.org/10.4236/aid.2017.74012

Received: September 30, 2017

Accepted: November 5, 2017

Published: November 8, 2017

Copyright (ङ 2017 by authors and Scientific Research Publishing Inc. This work is licensed under the Creative Commons Attribution International License (CC BY 4.0).

http://creativecommons.org/licenses/by/4.0/

(c) (i) Open Access

\begin{abstract}
Acute-on-chronic liver failure (Acute-on-chronic liver failure, ACLF) is acute liver function decompensation on the basis of chronic liver disease. The progression of ACLF develops from advanced phase, plateau phase to remission phase. The pathophysiological basis of ACLF in different phases is various. In advanced phase, immune imbalance and systemic inflammatory reaction plays key roles. In this study, we try to assess the association between expression of NK cells and its receptors and prognosis of patients with ACLF in advanced phase. A total of 35 inpatients with HBV acute-on-chronic liver failure in advanced phase were recruited. They were divided into case group $(\mathrm{n}=18)$ and control group $(n=17)$ according to whether the patients was dead in the 12 weeks. PBMC were detected for the frequency and expression of NK cell receptors by flow cytometric analysis. Our results demonstrated that patients who died had lower expression of NK cells and inhibitory receptor KIR3DL1, higher levels of FASL. During 12-week follow-up in those case alive, we found that NK cells increased, while expression of FASL decreased. High short-term mortality of ALCF was associated with NK cell, especially related to KIR3DL1 and FASL $\left(P_{N K}=0.036, P_{\text {KIRZDL1 }}=0.0265, P_{\text {FasL }}=0.0008\right)$.
\end{abstract}

\section{Keywords}

HBV, ACLF, NK Cell, Flow Cytometry

\section{Introduction}

Acute-on-chronic liver failure (ACLF) results in multiorgan failure and high 
short-term mortality and causes a series of symptoms including jaundice, coagulopathy, ascites, hemorrhage, cholestasis, hepatic encephalopathy, hepatorenal syndrome [1]. In China, the most common cause for liver failure is hepatitis B virus infection. The progression of ACLF can be divided into several stages: advanced phase, plateau phase and remission phase. In different phase, patients undergo various pathophysiology [2]. In advanced phase, immune damage and hypoxic-ischemic damage may be dominant [3].

Currently, no criteria are available for the precise definition of different phases. According to whether their total bilirubin within 7 days of average daily rise is $\geq 17.1 \mathrm{umol} / \mathrm{L}$ or not, we differentiated patients with ACLF into advanced phase and not-advanced phase.

NK cells are important immune cells which are closely related to antitumor, antiviral and immune regulation in vivo [4] [5]. In the early stage of acute HBV infection, the cytotoxicity of NK cells increases, IL-2 levels rise, both play the clearance of the virus. In chronic hepatitis B, cirrhosis, hepatocellular carcinoma patients, the number and activity of NK cells were suppressed [6]. The persistence of HBV may be associated with viral suppression of NK cell as the representative of the innate immune system.

In this study, we try to assess the association between expression of NK cells and its receptors and prognosis of patients with ACLF in advanced phase. Dynamic observation of immunological characterization of natural killer cell was tested in those case alive.

\section{Materials and Methods}

\subsection{Study Participants}

This study was a retrospective research. All patients were hospitalized in The Third Affiliated Hospital of Sun Yat-Sen University, a large tertiary comprehensive hospital with discharged patients more than forty thousand per year. A total of 35 inpatients with HBV related acute-on-chronic liver failure (HBV-ACLF) were recruited from October 2010 to September 2012 from our department. All procedures performed in studies involving human participants were in accordance with the ethical standards of the institutional and/or national research committee and with the 1964 Helsinki declaration and its later amendments or comparable ethical standards. Informed consent was obtained from all individual participants included in the study. The diagnoses of chronic hepatitis B and liver failure were based on consensus recommendations of APASL 2009 [7]. All patients' total bilirubin within 7 days of average daily rise is $\geq 17.1 \mathrm{umol} / \mathrm{L}$. Patients were excluded if they had some special conditions such as concomitant autoimmune diseases, important organ dysfunctions not due to ACLF malignancies, pregnancy and lactation, excessive alcohol drinking within 6 months. They were divided into case group $(\mathrm{n}=18)$ and control group $(\mathrm{n}=17)$ according to whether the patients was dead in the 12 weeks follow-up. The cause of death might be related to different complications occurred during ACLF pro- 
gression. Blood samples were collected from patients in different time point. Peripheral blood mononuclear cells (PBMC) were isolated, and percentage of natural killer cell, inhibitory NK receptors, activating NK receptors and cytotoxicity associated factors were determined by flow cytometry analysis. There were no significant differences at baseline level of two group patients, after comparison of clinical and biochemical indexes (Table 1).

\subsection{Flow Cytometric Analysis}

Peripheral blood mononuclear cells (PBMC) were isolated from fresh heparinized blood from patients on baseline, week 2, week 4, week 8, week 12 via Ficoll-Paque (Stemcell Technologies Inc., Vancouver, BC, Canada) separation. Cells were washed twice with phosphate-buffered saline (PBS) and suspended in RPMI-1640 medium supplemented with $10 \%$ heat-inactivated fetal calf serum (FCS) for further use. The expression of activating NK receptors NCRs (NKp30, NKp46) and Granzyme B on human peripheral NK cell (CD3-CD16+CD56+ and CD3-CD16-CD56+) was analyzed by the following mAbs: V450-conjugated anti-CD3, APC-CY7-conjugated anti-CD16, PERCP-CY5.5-conjugated anti-CD56, PE-CY7-conjugated anti-hNKp46, A488-conjugated anti-hNKp30, Alexa Flour 700-conjugated anti-Granzyme B, which were obtained from BD Bioscience (SanJose, CA). The expression of inhibitory NK receptors (NKG2D, KIR2DL1, KIR2DL3, KIR3DL1) and NKG2A was analyzed by the following mAbs: PE-conjugated anti-hNKG2A, APC-conjugated anti-hNKG2D, Flour MAb-conjugated anti-hKIR2DL1, PE-conjugated anti-hKIR1DL3, APC-conjugated anti-hKIR3DL1, which were obtained from R\&D Systems (Minneapolis, MN). FITC-conjugated anti-Perforin and PE-conjugated anti-FASL were obtained from eBioscience (San Diego, CA). Stained cells were analyzed by the BD FACSCalibur (BD Bioscience). The lymphocyte gate was established using forward and side scatter parameters. The positive gate was set using isotype controls (all from BD Bioscience). The percentages of NK cells expressing activating receptors, inhibitory receptors, FASL, Perforin, and Granzyme B were determined individually according to instructions, analyzed using FlowJo software.

\subsection{Statistical Analysis}

All data were analyzed using Windows software SPSS version 20.0. Data of clinical and biochemical features were expressed as frequency and mean \pm standard

Table 1. Comparison of clinical, biochemical and virologic characteristics of patients in the case group and control group.

\begin{tabular}{ccccccccccc}
\hline & $\mathrm{n}$ & $\begin{array}{c}\text { Gender } \\
(\text { male/female })\end{array}$ & $\begin{array}{c}\text { Age } \\
(\text { years })\end{array}$ & $\begin{array}{c}\text { ALT } \\
(\mathrm{U} / \mathrm{L})\end{array}$ & $\begin{array}{c}\text { ALB } \\
(\mathrm{g} / \mathrm{L})\end{array}$ & $\begin{array}{c}\text { Total bilirubin } \\
(\text { umol/L) }\end{array}$ & $\begin{array}{c}\text { PTA } \\
(\%)\end{array}$ & $\begin{array}{c}\text { HBeAg } \\
(+/-)\end{array}$ & $\begin{array}{c}\text { HBV-DNA } \\
\text { loads }(\mathrm{lgIU} / \mathrm{ml})\end{array}$ & $\begin{array}{c}\text { MELD } \\
\text { score }\end{array}$ \\
\hline Case & 18 & $18 / 0$ & $38.7 \pm 9.6$ & $137.0 ; 109.3,180$ & $33.6 \pm 5.2$ & $523.2 \pm 126.4$ & $35.5 \pm 11.5$ & $6 / 12$ & $3.8 ; 2.1,8.0$ & $27.1 \pm 4.1$ \\
Control & 17 & $16 / 1$ & $41.2 \pm 9.5$ & $171.0 ; 144.0,207.4$ & $35.2 \pm 5.4$ & $472.4 \pm 171.9$ & $36.1 \pm 9.9$ & $5 / 12$ & $3.4 ; 2.0,4.4$ & $25.6 \pm 3.8$ \\
$\chi^{2}$ or $F$ & & 1.059 & -0.121 & -1.388 & 0.843 & 1.07 & 0.087 & 0.062 & -1.098 & 0.000 \\
$P$ & & 0.486 & 0.926 & 0.186 & 0.365 & 0.352 & 0.765 & 0.803 & 0.271 & 0.995 \\
\hline
\end{tabular}


deviation, and compared using the chi-square and tests. The frequency was compared using Chi-square, and the quantitative data was compared using $t$ tests. Spearman's correlation was used between variables. One-way ANOVA analysis was used between quantitative data. $P$-values $<0.05$ were considered statistically significant.

\section{Result}

\subsection{Lower Levels of NK Cells and Inhibitory Receptor KIR3DL1 in Case Group}

The comparison of NK cells showed lower levels in case group compared to control group $(Z=26, P=0.036)$. There were no markedly differences between case group and control group, after comparison of activating NK receptors NKG2D, NKp30, NKp46 $(P>0.05)$. But the percentage of inhibitory NK receptor KIR3DL1 in case group was lower than in control group $(Z=17, P=0.0265)$. Other inhibitory NK receptors NKG2A, KIR2DL1, KIR2DL3 had no significant differences $(P>0.05$; Figure 1$)$.

\subsection{Higher Levels of FASL in Case Group}

The expression of FASL in case group was much higher than that in control group $(Z=0.00, P=0.0008)$. Significant deviations were not observed in Perforin and Granzyme B $(P>0.05$; Figure 2$)$.

\subsection{Increased NK Cells Expression during 12-Week Follow-up in Control Group}

Dynamic observation of immunological characterization of NK cells in case

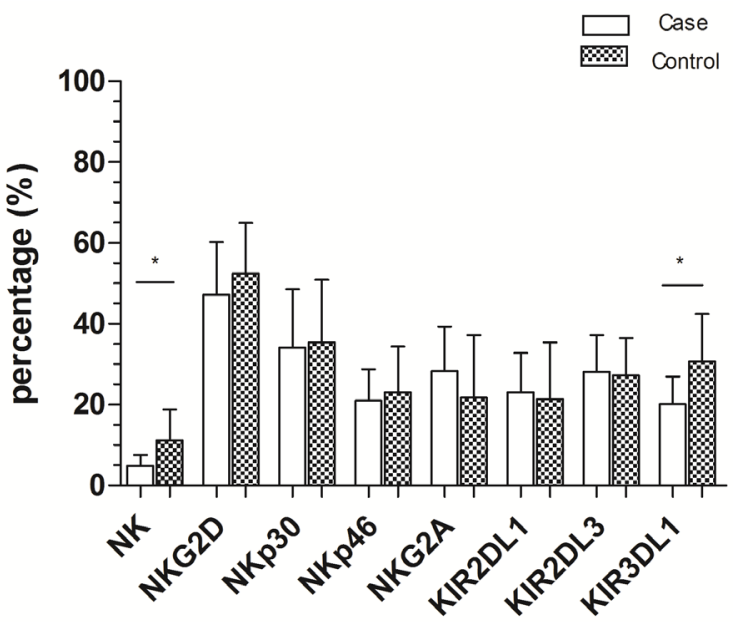

Figure 1. Lower levels of NK cells and inhibitory receptor KIR3DL1 in case group. 1) *: The percentage of NK cells in case group patients was lower than that in Control patients $(Z=26, P=0.036) ; 2) *$ : The percentage of inhibitory NK receptor KIR3DL1 in case group was lower than in control group $(Z=17, P=0.0265)$. There were no significantly differences in activating NK receptors NKG2D, NKp30, NKp46, inhibitory NK receptors NKG2A, KIR2DL1, KIR2DL3, $P>0.05$. 


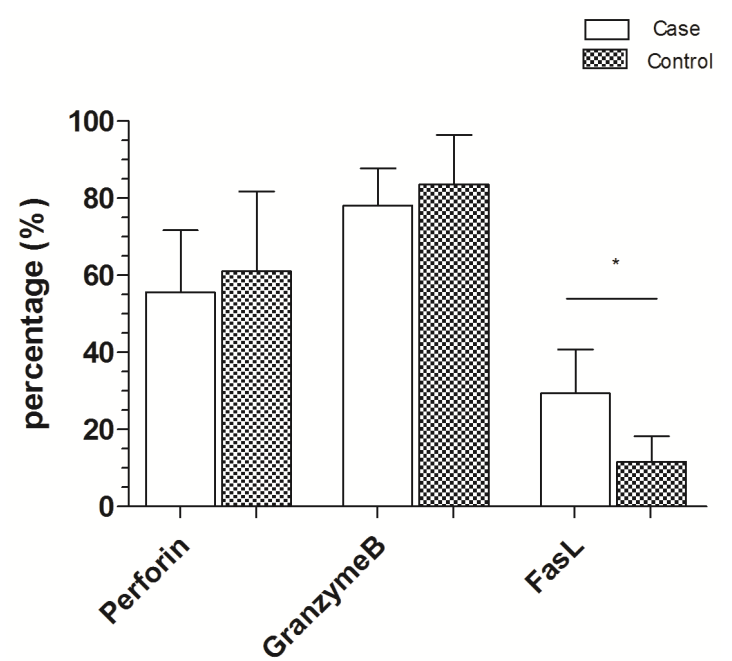

Figure 2. Higher levels of FASL in case group. ${ }^{*}$ : The expression of FASL in case group was much higher than that in control group $(Z=22.5, P=0.0008)$. Such difference was not observed in Perforin and Granzyme B $(P>0.05)$.

group showed that the percentage of NK cells gradually increased during the follow-up, had statistical difference in baseline and 12-week $(Z=-2.06, P=$ 0.039). The inhibitory receptor KIR3DL1 gained fluctuation during follow-up, but had no statistical difference in each time point $(P>0.05)$. The expression of FASL decreased during 12 weeks observation, though all-time point had no significant differences $(P>0.05$; Figure 3$)$.

\section{Discussion}

The progression of ACLF can be divided into several stages: advanced phase, plateau phase, and remission phase. The pathophysiological basis of ACLF in different stages is various. Immune imbalance and systemic inflammatory reaction plays a key role in the development of liver failure. Immune damage may be dominant in early advanced phase, then hypoxic-ischemic damage and endotoxin begin to take effect [8]. During the progress of ACLF, immunosuppression played a major role in plateau phase. When it comes to remission phase, immune reconstruction and rebalance begin to work [9]. According to whether their total bilirubin within 7 days of average daily rise is $\geq 17.1 \mu \mathrm{mol} / \mathrm{L}$, we differentiated patients with ACLF into advanced phase and investigate their relationship between immunological situation and prognosis.

Some previous studies had documented NK cells activated is related with HBV-ACLF [10] [11] [12]. Liver injury induced by HBV mainly depends on the host immunological response to the virus. Immune injury caused by HBV is mainly in cellular immunity, including dendritic cells, NK cells and CTL cells [13] [14]. Some studies had found that activity of NK cells in patients with ACLF was significantly increased, the progression of disease was associated with the increase of NKp30 receptor and NKp46, and liver injury mediated by NK cells through FAS/FASL and NKG2D/NKG2D ligand pathway [15] [16]. Our results 
NK

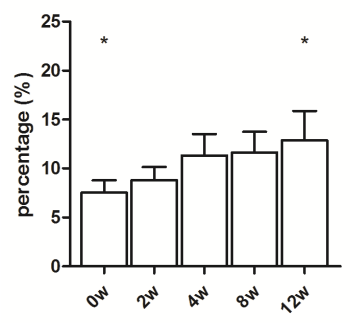

KIR3DL1

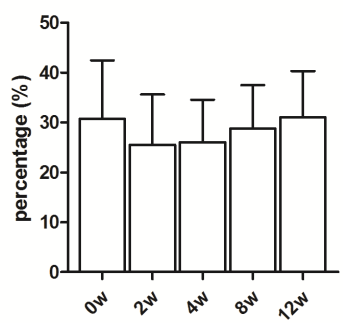

FasL

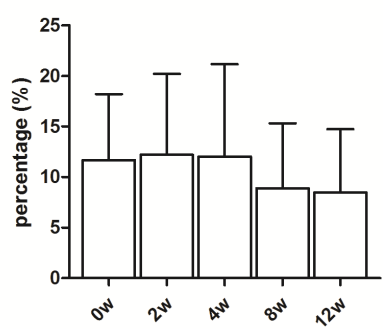

Figure 3. Increased NK cells expression during 12-week follow-up in control group. *: The percentage of NK cells gradually increased during the follow-up, had statistical difference in baseline and 12 -week $(Z=-2.06, P=0.039)$. The inhibitory receptor KIR3DL1 gained fluctuation during follow-up, but had no statistical difference in each time point $(P>0.05)$. The expression of FASL decreased during 12 weeks observation, though all-time point had no significant differences $(P>0.05)$.

demonstrated that patients who died of liver failure in advanced phase had lower expression of NK cell and inhibitory receptor KIR3DL1, while higher levels of FASL were observed. Considering the occurrence of ACLF, patients' livers were undergoing inflammatory imbalance and pro-inflammatory stat, leading to severe inflammation of the liver and necrosis of hepatocytes. As for the expression of inhibitory receptor KIR3DL1 decreased and FASL increased, cytotoxicity of NK cells enlarged significantly. Totally, activated NK cells lead to critical liver damage and poor prognosis. Then we dynamically observed NK cells, KIR3DL1 and FASL in control group. Those patients alive had increased NK cells, fluctuated KIR3DL1 expression, while expression of FASL decreased. It seems that recovery patients tend to regeneration of liver cells, reduce local inflammation and regulate local immune. Because of small numbers of cases involved, partial data no significant difference. The immunological changes of NK cells in advanced phase of ALCF provides a basis for further study of the occurrence and development mechanism of liver failure.

\section{Conclusions}

In summary, results from this study demonstrated that prognosis of patients with ACLF in advanced group had higher expression of NK associated immunological situation, including more NK cells gathered in liver, lower expression of inhibitory receptor KIR3DL1 and higher expression of FASL. If patients stayed alive during progress of ACLF, their immunological imbalance tended to recover and reconstruct. We think NK cells play a major role during ACLF process, and its function is mainly passed by FAS/FASL pathway which causes hepatocytes apoptosis and necrosis.

The limitations of this study include the small number of samples, no liver immunopathogenic, especially NK cells expression in the liver and single center study. Some questions need to be worked out, such as the cytokines involved in progression of ACLF, immunological changes in plateau phase and immunological intervention during advanced phase. A deeper level of immune investigation 
is also needed. So, further research requires more investigation and continuous cooperation between clinicians, researchers, and patients.

\section{Acknowledgements}

This research was supported by the National Science and Technology Major Project (2012ZX10002004 and 2012ZX10002007), National Basic Research Program of China (2012CBA01302, 2010CB945400), Guangzhou major project in collaborative innovation of industry (1561000157), the Natural Science Foundation of Guangdong Province (9151040701000019, S2013030013305), Key Scientific and Technological Program of Guangzhou City (201300000089, 2010U1-E00551) and the Fund for Guangdong translational medicine public platform.

\section{Conflicts of Interest}

We declare that we have no conflict of interest.

\section{References}

[1] Sarin, S.K., Kedarisetty, C.K., Abbas, Z., et al. (2014) Acute-on-Chronic Liver Failure: Consensus Recommendations of the Asian Pacific Association for the Study of the Liver (APASL) 2014. Hepatology International, 8, 453-471.

https://doi.org/10.1007/s12072-014-9580-2

[2] Wang, X., Sarin, S.K. and Ning, Q. (2015) Definition of ACLF and Inclusion Criteria for Extra-Hepatic Organ Failure. Hepatology International, 9, 360-365. https://doi.org/10.1007/s12072-015-9637-x

[3] Mikolasevic, I., Milic, S., Radic, M., et al. (2015) Clinical Profile, Natural History, and Predictors of Mortality in Patients with Acute-on-Chronic Liver Failure (ACLF). Wiener klinische Wochenschrift, 127, 283-289. https://doi.org/10.1007/s00508-015-0707-9

[4] Leavy, O. (2012) Maturation and Function of NK Cells. Nature Reviews Immunology, 12, 150.

[5] Rehermann, B. (2015) Natural Killer Cells in Viral Hepatitis. Cellular and Molecular Gastroenterology and Hepatology, 1, 578-588. https://doi.org/10.1016/j.jcmgh.2015.09.004

[6] Sun, C., Sun, H., Zhang, C. and Tian, Z. (2015) NK Cell Receptor Imbalance and NK Cell Dysfunction in HBV Infection and Hepatocellular Carcinoma. Cellular \& Molecular Immunology, 12, 292-302. https://doi.org/10.1038/cmi.2014.91

[7] Sarin, S.K., Kumar, A., Almeida, J.A., et al. (2009) Acute-on-Chronic Liver Failure: Consensus Recommendations of the Asian Pacific Association for the Study of the Liver (APASL). Hepatology International, 3, 269-282. https://doi.org/10.1007/s12072-008-9106-x

[8] Wasmuth, H.E., Kunz, D., Yagmur, E., et al. (2005) Patients with Acute on Chronic Liver Failure Display "Sepsis-Like” Immune Paralysis. Journal of Hepatology, 42, 195-201. https://doi.org/10.1016/j.jhep.2004.10.019

[9] Antoniades, C.G., Berry, P.A., Wendon, J.A. and Vergani, D. (2008) The Importance of Immune Dysfunction in Determining Outcome in Acute Liver Failure. Journal of Hepatology, 49, 845-861. https://doi.org/10.1016/j.jhep.2008.08.009

[10] Liu, F., Duan, X., Wan, Z., et al. (2016) Lower Number and Decreased Function of 
Natural Killer Cells in Hepatitis B Virus Related Acute-on-Chronic Liver Failure. Clinics and Research in Hepatology and Gastroenterology, 40, 605-613. https://doi.org/10.1016/j.clinre.2016.01.004

[11] Zheng, Q., Zhu, Y.Y., Chen, J., et al. (2015) Activated Natural Killer Cells Accelerate Liver Damage in Patients with Chronic Hepatitis B Virus Infection. Clinical \& EXperimental Immunology, 180, 499-508. https://doi.org/10.1111/cei.12597

[12] Li, X., Zhang, M., Liu, J., et al. (2016) Intrahepatic NK Cells Function Suppressed in Advanced Liver Fibrosis. European Journal of Clinical Investigation, 46, 864-872. https://doi.org/10.1111/eci.12669

[13] Dong, X., Gong, Y., Zeng, H., et al. (2013) Imbalance between Circulating CD4+ Regulatory $\mathrm{T}$ and Conventional $\mathrm{T}$ Lymphocytes in Patients with HBV-Related Acute-on-Chronic Liver Failure. Liver International, 33, 1517-1526.

[14] Zhang, G.L., Xie, D.Y., Lin, B.L., et al. (2013) Imbalance of Interleukin-17-Producing CD4 T Cells/Regulatory T Cells Axis Occurs in Remission Stage of Patients with Hepatitis B Virus-Related Acute-on-Chronic Liver Failure. Journal of Gastroenterology and Hepatology, 28, 513-521. https://doi.org/10.1111/jgh.12082

[15] Zou, Y., Chen, T., Han, M., et al. (2010) Increased Killing of Liver NK Cells by Fas/Fas Ligand and NKG2D/NKG2D Ligand Contributes to Hepatocyte Necrosis in Virus-Induced Liver Failure. Journal of Immunology, 184, 466-475. https://doi.org/10.4049/jimmunol.0900687

[16] Okazaki, A., Hiraga, N., Imamura, M., et al. (2012) Severe Necroinflammatory Reaction Caused by Natural Killer Cell-Mediated Fas/Fas Ligand Interaction and Dendritic Cells in Human Hepatocyte Chimeric Mouse. Hepatology, 56, 555-566. https://doi.org/10.1002/hep.25651 